\title{
INDUCED REPRESENTATIONS OF NONLOCALLY COMPACT GROUPS
}

\author{
P. Gibilisco
}

\section{0 . Introduction}

Induced representations were introduced by Frobenius in 1898 for finite groups, and by Mackey in 1949 for locally compact separable groups. The main result of the theory is the imprimitivity theorem characterizing induced representations as those which have an associated imprimitivity system. A number of proofs of the imprimitivity theorem can be found in the literature [1-5]. The proof given by Ørsted in 1979 [6] seems to be the shortest and simplest (see also [7]).

Since the theory of representations of locally compact groups can be seen as a special case of the theory of *-representations of involutory Banach algebras, the theory of induced representations has been generalized in the *-algebraic direction by many authors [8-13]. Also, in these contexts, imprimitivity theorems have been proved. However, when pushed down to the group case, these generalizations are based on the hypothesis of local compactness.

Physical applications of the theory of induced representations for locally compact groups are well known (see [14]) and references therein). Actually, infinite dimensional groups arise in physics in at least four different contexts: fluid dynamics, gauge theory, general relativity, and quantum field theory. It is well known that in the case of infinite dimension one cannot use the hypothesis of local compactness, and therefore at first sight it seems impossible to apply the theory of induced representations for solving the ab. 're-mentioned problems.

Actually, the inducing process has been carried out in many cases (at different levels of rigor) without the assumption of local compactness (see $[4,15-30])$. It was shown (see $[16,18]$ ) that in special cases (as in the classical theory) the equivalence between irreducibility of the inducing representation and that of the associated imprimitivity system occurs.

Despite these facts there is no attempt in the mathematical literature (to the author's knowledge) to prove the main result of Mackey's theory, namely the imprimitivity theorem, at least for the class of nonlocally compact groups. In this paper, we prove such a theorem (see Theorem 4.1 below) in the case of a compact quotient.

In what follows we consider a separable topological group $G$ and a closed subgroup $K \subseteq G$ such that the quotient $M=G / K$ is a compact Hausdorff space and there exists a Borel cross section from $M$ to $G$. We show how to define an induced representation $V^{(L, m)}$ of $G$ once one has fixed a representation $L$ of $K$ and a $G$-quasi-invariant measure $m$ on $M$. The main result consists in the proof of the imprimitivity theorem under the above hypotheses. This result is based on the von Neumann direct integral decomposition theory for Hilbert spaces [31]. It is remarkable that, although the use of the Haar measure is forbidden by the hypotheses, the proof appears to be very simple. The scheme of the proof was described earlier in [18, 32].

The path groups, studied in $[16,18]$ (and, at a physical level of rigor, in [29, 30]), give examples of nonlocally compact groups satisfying the hypotheses of the theorem. It seems likely that this imprimitivity theorem applied to the case of path groups, together with Driver's classification of bundle-connection pairs

Centro Matematico V. Volterra, Dipartimento di Matematica, Università di Roma II, Via Orazio Raimondo 00173 Roma, Italy. Translated from Matematicheskie Zametki, Vol. 57, No. 3, pp. 350-358, March, 1995. Original article submitted December 28, 1994. 
[6], can be used to classify bundle-connection pairs as imprimitive representations of path groups over symmetry groups for the base manifolds. This problem will be discussed in a forthcoming paper.

\section{Preliminary notions}

In what follows, $G$ is a separable topological group and $K$ is a closed subgroup such that $M=G / K$ is a compact Hausdorff space. We assume that there exists a Borel measurable cross section $s$ for the canonical projection $\pi: G \rightarrow G / K$, i.e., a Borel measurable function $s: G / K \rightarrow G$ such that $(\pi \circ s)(x)=x$ for any $x \in M=G / K$. There are known criteria which guarantee the existence of such sections [11, 12]. All the Hilbert spaces are assumed to be complex and separable, and by a representation of a topological group we mean a continuous unitary representation in a Hilbert space. For the notion of measurable fields of Hilbert spaces, vector fields, direct integrals, decomposable operators, etc., we refer to [31]. We also use the following result ([31, p. 167, Proposition 4]).

Proposition 1.1. Let $M$ be a Borel space, $m$ a positive measure on $M$, and $x \rightarrow H_{x}$ a field of Hilbert spaces over $M$. Assume that $f_{n}(\cdot)$ is a sequence of vector fields possessing the following properties:

(i) the functions $x \rightarrow\left(f_{i}(x), f_{j}(x)\right)$ are measurable;

(ii) $f_{n}(x)$ is a total sequence in $H_{x}$ for any $x \in M$.

Then there exists exactly one measurable field structure on the family $\left\{H_{x}\right\}$ such that the fields $f_{n}(\cdot)$ are measurable.

\section{G-fields of Hilbert spaces and representations of subgroups}

For the materials of this section we refer to Ch. 9 of [33]. A field of Hilbert spaces over $M$ is a $G$-field if there exists a family of unitary operators $A_{x}^{g}: H_{x} \rightarrow H_{g x}$ such that for all $x \in M$ and for all $g, h \in G$ one has $A_{x}^{g h}=A_{h x}^{g} A_{x}^{h}$.

Two $G$-fields $\left\{H_{x}, A_{x}^{g}\right\},\left\{H_{x}^{\prime}, A_{x}^{\prime g}\right\}$ are isomorphic if there exists a family of unitary operators $B_{x}: H_{x} \rightarrow$ $H_{x}^{\prime}$ such that $B_{g x} A_{x}^{g}=A_{x}^{\prime g} B_{x}$.

Example 2.1. Let $K$ be a closed subgroup of $G$, and let $L$ be a representation of $K$ in the Hilbert space $H$. Then $K$ acts on the space $G \times H$ by the rule $(g, v) \rightarrow\left(g k, L_{k}^{-1} v\right)$. Let $E:=(G \times H) / K$ be the quotient space under this action, and let $M=G / K$. Then we have the surjection $\pi: E \rightarrow M$ defined by $[(g, v)] \rightarrow g K$. Put $H_{x}:=\pi^{-1}(x)$, then $H_{x}$ inherits a Hilbert space structure in the following way. Using the existence of a Borel section $s: G / K \rightarrow G$, we fix an element $g_{0} \in G$ such that $x=g_{0} K$. Then any element of $H_{x}$ can be written in a unique way as $\left[\left(g_{o}, v\right)\right], v \in H$. Thus, we obtain a natural identification of $H_{x}$ with $H$. This identification gives a bijection between vector fields in $\prod H_{x}$ and functions $f: M \rightarrow H$. Now let $h_{n}$ be a total sequence in $H$. Assume that $f_{n}(x):=h_{n}$ for any $x \in M$. It is easy to see that the sequence of vector fields corresponding to $f_{n}(\cdot)$ satisfies conditions (i) and (ii) of Proposition (1.1). Therefore, $\left\{H_{x}\right\}$ inherits a measurable field structure. We define the unitary operators $A_{x}^{g}: H_{x} \rightarrow H_{g x}$ by $A_{x}^{g}\left[\left(g^{\prime}, v\right)\right]:=\left[\left(g g^{\prime}, v\right)\right]$, where $x=g^{\prime} K$. In this way we have obtained a measurable $G$-field of Hilbert spaces. Note that by choosing a different section or a different total set one finds an isomorphic measurable $G$-field.

Suppose now that we have a transitive measurable $G$-field of Hilbert spaces $\left\{H_{x}, A_{x}^{g}\right\}$ over $M$. Let $K_{x_{o}}$ be the isotropy group of $x_{o} \in M$. Then we have a representation of $K_{x_{o}}$ on $H_{x_{0}}$.

We may construct the $G$-field of Hilbert spaces as we have done before. Let $\pi: G \times H_{x_{o}} / K_{x_{\circ}} \rightarrow G / K_{x_{\circ}}$ and $H_{x}{ }^{\prime}:=\pi^{-1}(x)$. Let us identify $M$ with $G / K_{x_{0}}$ by the rule $x \rightarrow g K_{x_{0}}$, where $g x_{0}=x$. Then the relation $g x_{0}=x$ implies $A_{x_{0}}^{g}: H_{x_{0}} \rightarrow H_{g x_{0}}=H_{x}$; therefore, if $v \in H_{x_{0}}$, then $A_{x_{0}}^{g} v \in H_{x}$. Hence, the mapping $[(g, v)] \in H_{x}{ }^{\prime} \rightarrow A_{x_{0}}^{g} v \in H_{x}$ gives an isomorphism $B_{x}: H^{\prime}{ }_{x} \rightarrow H_{x}$ between the two $G$-fields.

In this way we have a bijection between representations of closed subgroups $K$ of $G$ and measurable transitive $G$-fields of Hilbert spaces over $M=G / K$.

A smooth version of the content of this section can be found in the book of Wallach [34]. 


\section{Induced representations and imprimitivity systems}

Let $L$ be a representation of $K^{\circ}$, and let $m$ be a G-quasi-intariant Borel measure on . 1/. As was shown in the preceding paragraph, using $L$ we can construct a measurable $G$-field of Hilbert spaces $\left\{H_{x}, A_{x}^{g}\right\}$ over $M$. We now define the direct integral $\mathcal{H}:=\mathcal{H}^{(L, m)}:=\int_{M}^{\oplus} H_{x} d m(x)$ as the Hilbert space of square-integrable vector fields. Put $H_{x}^{g}:=H_{g x}$. It is easy to see that $\left\{H_{x}^{g}\right\}$ is a measurable field; therefore, we may consider the integral

$$
\mathcal{H}^{g}:=\int_{M}^{\oplus} H_{x}^{g} d m(x)
$$

We denote by $\frac{d m^{g}}{d m}(x)$ the Radon-Nykodim derivative of the measure $m(g \cdot)$ with respect to the measure $m(\cdot)$. Now let us define the operators $A_{g}, T_{g}: \mathcal{H} \rightarrow \mathcal{H}^{g}$ by

$$
\begin{aligned}
\left(T_{g} f\right)(x) & :=\left(\frac{d m^{g}}{d m}(x)\right)^{1 / 2} f(g x) \quad f \in \mathcal{H} \\
A_{g} & :=\int_{M}^{\oplus} A_{x}^{g} d m(x) .
\end{aligned}
$$

The operator $A_{g}$ is unitary, since all the operators $A_{x}^{g}$ are unitary.

Moreover, we obtain for $T_{g}$,

$$
\begin{aligned}
\left\|T_{g} f\right\|_{\mathcal{H}^{g}}^{2} & =\int_{M}\left\|\left(T_{g} f\right)(x)\right\|_{\mathcal{H}_{x}^{g}}^{2} d m(x)=\int_{M}\left\|\left(\frac{d m^{g}}{d m}(x)\right)^{1 / 2} f(g x)\right\|_{\mathcal{H}_{g x}}^{2} d m(x)= \\
& =\int_{M}\|f(g x)\|_{\mathcal{H}_{g x}}^{2} \frac{d m^{g}}{d m}(x) d m(x)=\int_{M}\|f(x)\|_{\mathcal{H}_{x}}^{2} d m(x)=\|f\|_{\mathcal{H}^{\prime}}^{2} .
\end{aligned}
$$

Thus, $T_{g}$ is an isometry. It is easy to see that $T_{g}$ has an inverse. This implies that $T_{g}$ is surjective and, therefore, unitary.

Definition 3.1. The induced representation

$$
V_{g}^{(L, m)}: \mathcal{H}^{(L, m)} \rightarrow \mathcal{H}^{(L, m)}
$$

is defined by the relations

$$
V_{g}^{(L, m)}:=A_{g^{-1}}^{-1} T_{g^{-1}}
$$

Let $\psi \in L^{\infty}(M, d m)$. The operator $P(\psi):=P^{(L, m)}(\psi): \mathcal{H} \rightarrow \mathcal{H}$ is defined by

$$
\left(P^{(L, m)}(\psi) f\right)(x):=\psi(x) f(x) .
$$

In the same way we can define $P_{g}(\psi): \mathcal{H}^{g} \rightarrow \mathcal{H}^{g}$. Since $A_{g}$ is decomposable, it follows that

$$
A_{g} P(\psi)=P_{g}(\psi) A_{g} .
$$

By direct calculations we obtain

$$
V_{g}^{(L, m)} P^{(L, m)}(\psi)=P^{(L, m)}\left(\psi^{g}\right) V_{g}^{(L, m)}
$$

where $\psi^{g}(x):=\psi\left(g^{-1} x\right)$.

Definition 3.2. A transitive imprimitivity system based on $M$ is a triple $(V, P, \mathcal{H})$, where $V$ is a representation of $G$ in a Hilbert space $\mathcal{H}, G$ acts transitively on $M$, and $P$ is *-homomorphism from $C(M)$ to $\mathcal{B}(\mathcal{H})$ such that

$$
V_{g} P(\psi)=P\left(\psi^{g}\right) V_{g}
$$

Definition 3.3. A transitive imprimitivity system $(V, P, \mathcal{H})$ is called induced if it is unitary equivalent to a system of the form $\left(V^{(L, m)}, P^{(L, m)}, \mathcal{H}^{(L, m)}\right)$. 


\section{The imprimitivity theorem}

Theorem 4.1. Let $(V, P, \mathcal{H})$ be a transitive imprimitivity system for $G$, based on a compact space $M$. Then $(V, P, \mathcal{H})$ is induced.

Proof. Since

$$
V_{g} P(\psi)=P\left(\psi^{g}\right) V_{g}
$$

it follows that $K \operatorname{er} P$ is a $G$-invariant ideal of $C(M)$ and, therefore, is the zero ideal because of transitivity. This means that $P: C(M) \rightarrow P(C(M))$ is an isomorphism and, therefore, the spectrum of $P(C(M))$ is homeomorphic to the spectrum of $C(M))$, i.e., $M$.

Now let $\mathcal{A}$ be the weak closure of $P(C(M))$ in $\mathcal{B}(\mathcal{H})$. Since $\mathcal{H}$ is separable, $\mathcal{A}$ is $\sigma$-finite (see [31], p. 3) and, therefore, $M=S p(P(C(M))$ carries a bounded basic measure $m$ (see [31], p. 130). The decompositions theorem ([31], p. 233, Th. 1; p. 236, Th. 2) imply that $\mathcal{H}$ has an integral decomposition

$$
\int_{M}^{\oplus} \mathcal{H}_{x} d m(x)
$$

where the operators $P(\psi)$ can be identified with the operators $f(\cdot) \rightarrow \psi(\cdot) f(\cdot)$ for $f \in \mathcal{H}$. Furthermore, $P$ extends to $L^{\infty}(M, d m)$, and $P\left(L^{\infty}(M, d m)\right)$ coincides with $\mathcal{A}$. If $S, T$ belong to $\mathcal{B}(\mathcal{H})$, then the mappings $B \rightarrow S B, B \rightarrow B T$ are weakly continuous (see [31], p. 35). Assume that $\psi \in L^{\infty}(M, d m)$. Since $P(\psi)$ is the weak limit of $P\left(\psi_{n}\right)$ for some sequence $\psi_{n} \in C(M)$, it follows that for any $g \in G$ we have

$$
V_{g} P(\psi)=V_{g} \lim P\left(\psi_{n}\right)=\lim V_{g} P\left(\psi_{n}\right)=\lim P\left(\psi_{n}^{g}\right) V_{g}=\left(\lim P\left(\psi_{n}^{g}\right)\right) V_{g}=P\left(\psi^{g}\right) V_{g}
$$

This irpplies that the measure $m(\cdot)$ is $G$-quasi-invariant. In fact,

$$
0=n(E) \Leftrightarrow 0=P\left(\chi_{E}\right) \Leftrightarrow 0=V_{g} P\left(\chi_{E}\right) V_{g}^{-1}=P\left(\chi_{E}^{g}\right) \Leftrightarrow \Leftrightarrow P\left(\chi_{g} E\right)=0 \Leftrightarrow m(g E)=0 .
$$

Now let $\mathcal{H}^{g}$ and $T_{g}$ be defined by $\left(3^{*}\right)$ and $\left(3^{* *}\right)$, respectively. Recall that $P_{g}(\psi): \mathcal{H}^{g} \rightarrow \mathcal{H}^{g}$ is defined by $\left(\left(P_{g} \psi\right) f\right)(x)=\psi(x) f(x)$ for $f(\cdot) \in \mathcal{H}^{g}$. Then we obtain for $h \in G$,

$$
\begin{aligned}
\left(T_{g^{-1}} P\left(\psi^{h}\right) f\right)(x) & =\left(T_{g^{-1}}\left(\psi\left(h^{-1} \cdot\right) f(\cdot)\right)(x)=\left(\frac{d m^{g^{-1}}}{d m}(x)\right)^{1 / 2} \psi\left(h^{-1} g^{-1} x\right) f\left(g^{-1} x\right)=\right. \\
& =\psi\left((g h)^{-1} x\right)\left(T_{g^{-1}} f\right)(x)=\psi^{g h}(x)\left(T_{g^{-1}} f\right)(x)=\left(\left(P_{g}\left(\psi^{g h}\right) T_{g^{-1}}\right) f\right)(x)
\end{aligned}
$$

i.e.,

$$
T_{g^{-1}} P\left(\psi^{h}\right)=P_{g}\left(\psi^{g h}\right) T_{g^{-1}}
$$

Define the operator $A_{g^{-1}}: \mathcal{H} \rightarrow \mathcal{H}^{g^{-1}}$ by

$$
A_{g^{-1}}:=T_{g^{-1}} V_{g^{-1}} \quad g \in G \text {. }
$$

Then $A_{g^{-1}}$ is unitary and

$$
A_{g^{-1}} P(\psi)=T_{g^{-1}} V_{g^{-1}} P(\psi)=T_{g^{-1}} P\left(\psi^{g^{-1}}\right) V_{g^{-1}}=P_{g}\left(\psi^{g g^{-1}}\right) T_{g^{-1}} V_{g^{-1}}=P_{g}(\psi) A_{g^{-1}}
$$

Therefore (see [31], p. 187, Th. 1), $A_{g^{-1}}$ is decomposable as

$$
A_{g^{-1}}=\int_{M}^{\oplus} A_{x}^{g^{-1}} d m(x)
$$


where $A_{x}^{g}: H_{x} \rightarrow H_{x}^{g}$ are unitary operators.

Fix elements $g, h \in G, x \in M$. Let $f$ be an arbitrary element of $\mathcal{H}$. Define $z:=g h x$ so that $h x=g^{-1} z$. Then we have

$$
\begin{aligned}
A_{x}^{g h} f(x) & =A_{h^{-1} g^{-1} z}^{g h} f\left(h^{-1} g^{-1} z\right)=A_{(g h)^{-1} z}^{g h}\left(T_{(g h)^{-1}} f\right)(z)=\left(V_{g h} f\right)(z) \\
& =\left(V_{g}\left(\left(V_{h} f\right)(\cdot)\right)\right)(z)=\left(V_{g}\left(A_{h^{-1}(\cdot)}^{h}\left(T_{h^{-1}} f\right)(\cdot)\right)\right)(z)=\left(V_{g}\left(A_{h^{-1}(\cdot)}^{h} f\left(h^{-1} \cdot\right)\right)\right)(z) \\
& =A_{\left(g^{-1} z\right)}^{g}\left(A_{h^{-1}\left(g^{-1} z\right)}^{h} f\left(h^{-1} g^{-1} z\right)\right)=A_{h x}^{g}\left(A_{x}^{h} f(x)\right) .
\end{aligned}
$$

Since $f$ is arbitrary, we conclude that $\forall g, h \in G$ and $\forall x \in M$ the following relation holds:

$$
A_{x}^{g h}=A_{h x}^{g} A_{x}^{h}
$$

Therefore, $\left\{H_{x} ; A_{x}^{g}\right\}$ is a $G$-field, and $V_{g}$ has the standard form

$$
V_{g}:=A_{g^{-1}}^{-1} T_{g^{-1}}
$$

This completes the proof.

I express my deep gratitude to Professor Luigi Accardi for his assistance and encouragement.

\section{REFERENCES}

1. L. H. Loomis, "Positive definite functions and induced representations," Duke Math. J., 27, 569-580 (1960).

2. G. W. Mackey, "Imprimitivity for representations of locally compact groups. I," Proc. Natl. Acad. Sci. USA, 35, 537-545 (1949).

3. G. W. Mackey, "Unitary representations of group extensions. I," Acta Math., 99, 265-311 (1958).

4. R. Loll, "A new quantum representation for the canonicil gravity and SU(2) Yang-Mills theory," Nucl. Phys. B, 350, 831-860 (1991).

5. R. J. Blattner, "Positive definite measures," Proc. Am. Math. Soc., 14, 423-428 (1963).

6. P. E. T. Jorgensen, Operators and Representation Theory, North-Holland, Amsterdam (1988).

7. B. Ørsted, "Induced representation and a new proof of the imprimitivity theorem," J. Funct. Anal., 31, 355-359 (1979).

8. J. M. G. Fell, "An extension of Mackeys method to Banach *-algebraic bundles," Memoirs Am. Math. Soc., 90 (1969).

9. J. M. G. Fell and R. S. Doran, Representations of *-Algebras, Locally Compact Groups, and Banach *-Algebraic Bundles. Vols. 1, 2, Academic Press (1988).

10. P. Green, "The local structure of twisted covariance algebras," Acta Math., 140, 191-250 (1978).

11. K. Mansfield, "Induced representations of crossed products by coactions," J. Funct. Anal., 97, 112-161 (1991).

12. M. A. Rieffel, "Induced representation of $C^{*}$-algebras," Adv. Math., 13, 176-257 (1974).

13. Takesaki, "Covariant representation of $C^{*}$-algebras and their locally compact automorphism groups," Acta Math., 119, 273-303 (1967).

14. A. O. Barut and R. Racka, Theory of Group Representations and Applications, World Sci. (1986).

15. L. Accardi, "The Weyl-Schrödinger representation on curved space and functional integrals on the connections," in: Proceedings of the X Italian Relativity Conference, World Sci. (to appear).

16. L. Accardi and P. Gibilisco, "The Schrödinger representation on Hilbert bundles," in: Probabilistic Methods in Mathematical Physics, Siena, 1991 (F. Guerra, M. I. Loffredo, and C. Marchioro, eds.), World Sci. (1992). 
17. A. B. Borisov, "The unitary representation of the Diff $R^{N}$ group," J. Phys. A: Math. Gen., 12, 1625 (1979).

18. P. Gibilisco, "Rappresentazioni indotte di gruppi di cammini e trasporti paralleli," Ph. D. Thesis [in Italian], Università degli studi di Roma "La Sapienza" (1992).

19. L. Girardello and G. Parravicini, "Some remarks on the representations of infinite parameter groups and of the BMS group," in: Group theoretical methods in physics (Proc. III Intern. Colloq., Centre Phys. Theor., Marseille) (1974).

20. G. A. Goldin, R. Menikoff, and D. H. Sharp, "Particle statistics from induced representation of a local current group," J. Math. Phys., 21, 650 (1980).

21. G. A. Goldin, R. Menikoff, and D. H. Sharp, "Induced representation of the group of diffeomorphisms," J. Phys., 16, 1827-1833 (1983).

22. G. A. Goldin, R. Menikoff, and D. H. Sharp, "Diffeomorphism groups, gauge groups, and quantum theory," Phys. Rev. Lett., 51, 25 (1983).

23. G. A. Goldin, R. Menikoff, and D. H. Sharp, "Diffeomophism groups and quantized vortex filaments," Phys. Rev. Lett., 58, 2162 (1987).

24. G. A. Goldin and D. H. Sharp, "Particle spin from representations of the diffeomorphism group," Comm. Math. Phys., 92, 217-228 (1983).

25. C. J. Isham, "Topological and global aspects of quantum theory," in: Relativity, Groups and Topology. Vol II, Les Houches, 1983 (B. S. DeWitt and R. Stora, eds.), North Holland, Amsterdam (1984).

26. C. J. Isham and A. C. Kaukas, "A group theoretical approach to the canonical quantization of gravity. I. Construction of the canonical group," Class. Quantum Grav., 1, 621-632 (1984).

27. C. J. Isham and A. C. Kaukas, "A group theoretical approach to the canonical quantization of gravity. II. Unitary representations of the canonical group," Class. Quantum Grav., 1, 633-650 (1984).

28. J. Marion, "Induction-unitarization process for some nonlocally compact topological groups," Universität Bielefeld, Preprint BiBoS, No. 471 (1991).

29. M. B. Menskii, Method of Induced Representations: Space-Time and Concept of Particle [in Russian], Nauka, Moscow (1976).

30. M. B. Menskii, "The path group and the interaction of quantum string," Sov. Phys. JETP, 63, No. 2 (1986).

31. J. Dixmier, "Von Neumann Algebras," North-Holland Math. Lib., 27 (1981).

32. P. Gibilisco, "The imprimitivity theorem for a class of nonlocally compact groups," in: Proceedings of the X Italian Relativity Conference, World Sci. (to appear).

33. G. W. Mackey, Unitary Representations in Physics, Probability, and Number Theory, Benjamin-Cummings Publ. Co. (1978).

34. N. R. Wallach, Harmonic Analysis on Homogeneous Space, Marcel Dekker, New York (1973). 